\title{
The Tribe Agrosteae of Japan, excluding The Genus Calamagrostis.
}

\author{
By
}

\section{Jisaburo Ohwi*}

Received July 15, 1941.

Trib. 1. Agrosteae Nees, Agrost. Brasil. (1829) 390, sens. str.

Gen. Agrostis Linn. Sp. Pl. (1753) 61 et Gen. ed. 5 (1754) 30.

1. Palea ca $1 / 2$ lemmatis aequilonga vel longior, antherae $2 / 3-4 / 5$ lemmatis aequantes.

2. Spiculae dimorphac, lemma aut muticum et glabrum, aut aristatum et margine pilosum. . . . . . dimorpholemma.

2. Spiculae omnes homomorphae, muticae vel rarissime aristatae, glabrae.

3. Rami paniculae post anthesin patentes, verticillis basi nudis.

4. Rami paniculae patentes, vix divaricati.

5. Ligula non super $2 \mathrm{~mm}$ longa. A. tenuis.

j. Ligula $3-\overline{\mathrm{j}} \mathrm{mm}$ longa.

A. gigantea.

4. Rami patentes, divaricati. A. divaricatissima.

3. Rami paniculae post anthesin erecti, non patentes.

4. Culmi basi repentes, panicula $5-10 \mathrm{~cm}$ longa. . . . . . . . . A. stolonifera.

4. Culmi elati, basi non repentes, panicula $10-20 \mathrm{~cm}$ longa. ........ palustris.

1. Palea obsoleta vel brevior quam $1 / 2$ lemmatis.

2. Lemma distincte brevius quam glumae.

3. Antherae $2 / 3-3 / 4$ lemmatis aequilongae, $0.8-1.5 \mathrm{~mm}$ longae, spiculae vulgo aristatae.

4. Spiculae $2.5-3 \mathrm{~mm}$ longae, arista fere supra basin vel in $1 / 3$ superne lemmatis inserta, distincte geniculata et torta, rami paniculae laeves vel scabriusculi.

5. Nervi laterales lemmatis omnes apice in setas rectas excurrentes. A. Hideoi.

5. Nervi laterales lemmatis non setuloso-excurrentes. .......... flaccida.

4. Spiculae $1.5-2.2 \mathrm{~mm}$ longae, arista ca. medio lemmatis inserta gracili vel obsoleta.

5. Planta montana vel pratensis, culmi elati, folia radicalia vix conspicua, rami paniculae post anthesin ascendentes tenues. ........... canina.

5. Planta alpina saxicola, $15-30 \mathrm{~cm}$ alta, folia radicalia conspicua, lamina conduplicata, panicula demum effusa, rami ascendentes vel saepius patentes, rigiduli strictuli.

A. Fukuyamae.

3. Antherae $1 / 2-1 / 4$ lemmatis aequilongae $0.4-0.7 \mathrm{~mm}$ longae, spiculae saepe muticae.

4. Spiculae aristatae (rarissime muticae ?) . ............. borealis.

4. Spiculae exaristatae.

5. Verticilli paniculae basi nudi, culmi 2-3-nodi.

6. Panicula post anthesin laxa effusa, ramis ascendentes vel patentes.

7. Panicula laxissima $30 \mathrm{~cm}$ longa, ramis scaberrimis capillaribus, apicem versus tantum spiculosis.

A. hiemalis.

7. Panicula vix longior quam $20 \mathrm{~cm}$, ramis scabris vel laevibus, a medio spiculosis.

* Botanical Institute, Kyoto Imperial University, Kyoto. 
8. Spiculate $1.2-1.5 \mathrm{~mm}$ longae, paniculae rami post anthesin patentes.

........... formosana.

8. Spiculae $2-2.5 \mathrm{~mm}$ longae, paniculae rami post anthesin ascendentes. . . . . . . . . . . . . . . . . .

6. Panicula post anthesin contracta angustata, rami crecti. . . A. clavata.

j. Verticilli paniculae a basi spiculosi, rami non patentes.

6. Culmi 2-3-nodi.

7. Gluma secunda brevi-acuminata, quam lemma paullo longior.

A. clavata var.

7. Gluma secunda longe-acuminata, conspicue longior quam lemma. ....

6. Culmi 4-5̃-nodi.

A. exarata.

2. Jemma quam glumae longius vel aequilongum.

A. arisan-montana.

Sp. 1. Agrostis dimorpholemma Orwi, hybr. (vel sp.?) nov.

Peremis (?), estolonifera, culmis erectis subtenuibus ca. $60 \mathrm{~cm}$ altis laevibus 3-4-nodis sub panicula vix scabris, foliorum laminis planis 1.5-2.5 $\mathrm{mm}$ latis, ligula truncata ad $1 \mathrm{~mm}$ longa, vaginis laevibus, panicula exserta effusa ovata ca. $15 \mathrm{~cm}$ longa laxa (demum paullo contracta?), ramis semiverticillatis ascendentibus supra medium spiculiferis parce scabris usque ad $10 \mathrm{~cm}$ longis, verticillis ca. 5 basi nudis, pedicellis apice clavatis, spiculis 2.5-3 mm longis viridulis et pallide sordide purpureo-suffusis, glumis late lanceolatis subaequalibus laevibus acutissimis, $1^{\text {ma }}$ carina superne $2 / 3$, $2^{\text {da }}$ superne $1 / 3$ scabris, flosculis dimorphis in eadem panicula, floseulis aut muticis, lemmate $1.7 \mathrm{~mm}$ longo trinervi oblongo mutico vel nervis lateralibus excurrente brevissime mucronulato, apice rotundo-erosulo, callo subglabro, palea $1 / 2-2 / 3$ lemmatis aequilonga, staminibus 3 , antheris $1.5 \mathrm{~mm}$ longis ; aut flosculis aristatis, lemmate ovato-oblongo $1.7 \mathrm{~mm}$ longo 3 - vel obsolete 5nervi, nervis marginalibus excurrentibus breviter biaristellato, dorso inferne parce adpresse piloso, nervo medio supra basin excurrente in aristam ca. 4 $\mathrm{mm}$ longam medio leviter geniculatam, callo pilis $1 / 4^{-1 / 5}$ lemmatis aequilongis barbato, processu rhachillae ovario breviore pareissime piloso, palea $1 / 2-3 / 5$ lemmatis aequilonga.

Nom. Jap. Bake-nukabo.

Hab. Hondo: Hakone-Asinoyu in Sagami (K. Hisaucin, Jul. 1930).

Sp. 2. Agrostis tenuis Sibth. Fl. Oxon. (1794) 36 ; Hiтchc. Man. Grass. U. S. (1935) 332 et 785; Philipps. in Journ. Linn. Soc. 51 (1937) 85 (var. hispida (Willo.) Phillipps. p. 86).

Agrostis capillaris (vix Linn. efr. Phillipps. l. c. 86, adnot.) With. Bot. Arr. ed. 2, 1 (1787) '73; Schischk. in·Komar. Fl. URSS. 2 (1934) 185.

Agrostis vulgaris With. l. c. ed. 3, 2 (1796) 132 ; Aschers. et Graebn. Synops. 2:1 (1899) 179 .

Agrostis pumila Linn. Mant. (1767) 31 (stat. deform.).

Nom. Jap. Ito-konukagusa. 
Hab. Saghalien. Distr. Neg. bor. omn. part.

Sp. 3. Agrostis gigantea Roth, Fl. Germ. 1 (1788) 31 ; Phillipis. 1. e. (1937) 90 (var. dispar (Michx.) Phillirps. l. c. 93).

Agrostis repens Cur'r. Obs. Brit. Gram. ed. 2 (1790) 35.

Agrost is nigra Willo. Bot. Arr. ed. 3,2 (1796) 131; Aschers. et Graebn. Synops. $2: 1$ (1899) 177.

Agrostis alba (non Linn.) Híche. Man. Grass. U. S. (1935) 331, f. 665. fide Phillipès. l. c.

Agrostis palustris (non Huds.) IIonda, Monogr. Poac. Japon. (1930) 186 , forsan p.p. minim.

Nom. Jap. Koro-konukagusa.

Hab. In Japonica rara (Kuriles austr. Saghal., Yezo, Hondo, Kiushiu). Distr. Reg. boreal.

Sp. 4. Agrostis divaricatissima Mez in Fedde Repert. 18 (1922) 4; OHw in Act. Phyt. et Geob. 6 (1937) 152; Kitag. in Rep. Inst. Sci. Res. Manch. 4 (1940) 75.

Agrostis stolonifera OHw in Bot. Mag. Tokyo 45 (1931) 183, non Lisx. Agrostis koreana OHwi in Fedde Repert. 36 (1934) 39.

Nom. Jap. Mansen-nukabo.

Hab. Korea. Distr. Manchuria.

Sp. 5. Agrostis stolonifera Linn. Sp. Pl. (1753) 62; Phillipps. I. c. (1937) 94 (var. stolonifera).

Agrostis coarctata Eнrн. ex Hoffm. Deutschl. Fl. ed. 2, 1 (1800) 37.

Agrostis maritima Lam. Encycel. Méth. 1 (1783) 61.

Nom. Jap. Hai-konukagusa.

Hab. Yezo vix spontanea, Nemuro, leg. S. Sakaguchi). Distr. Reg. bor.

Sp. 6. Agrostis palustris Huds. Fl. Angl. ed. 1 (1762) 27 ; Honda, Monogr. Poac. Japon. (1930) 186, saltem p.p. maxim.

Agrostis alba (vix Linn.) Auct. fl. Japon. etiam Hack. in Bull. Herb. Boiss. 7 (1899) 649 incl. f. coartata.

Agrostis stolonifera var. palustris (Huds.) FARw. in Mich. Acad. Rep. for 1919 (1920) 351; Philuipps. in Journ. Linn. Soc. 51 (1937) 98.

Agrost is polymorpha var. palustris (Huds.) Huds. l.c. ed. 2 (1778) 129. Agrostis nigra (vix Willd.) Hiтchc. Man. Gr. U. S. (1935) 330, f. 664. fide Phillipps. l. c.

Agrostis sylvatica Huds. Fl. Angl. ed. 1 (1762) 28, forma deformata. ?.Agrostis Osakai Honda in Bot. Mag. Tokyo 53 (1939) 51.

Nom. Jap. Konuka-gusa.

Hab. Kuriles austr. Saghal. Yezo, Hondo, Shikoku, Kiushiu, Korea. 
Distr. Reg. boreal. omn. part.

var. aristata Honda in Bot. Mag. Tokyo 40 (1926) 323.

Nom. Jap. Noge-konukagusa.

Hab. Hondo media (fide Honda, l. c.).

Sp. 7. Agrostis Hideoi Ohwi in Bot. Mag. Tokyo 44 (1930) 568.

Senisetum Hideoi (OHwi) Honda in Bot. Mag. Tokyo 46 (1932) 371 et 47 (1933) 146.

Agrostis heptaseta H. KoIDz. ex Honda ibid. (1933) 146, pro syll.

Heptaseta japonica H. KoIDz. ibid. 146, pro syn.

Nom. Jap. Yukikura-nukabo, Okayama-nukabo.

Hab. Hondo media in montibus.

Sp. 8. Agrostis flaccida Hack. in Bull. Herb. Boiss. 7 (1899) 649 ; Matsum. Ind. Pl. Japon. $2: 1$ (1905) 34; HondA, Monogr. Poac. Japon. (1930) 189.

Agrostis canina (non Linn.) Hack. l. c. (1899) 649; Honda, l. c. (1930) 188, p.p.

Agrostis debilis Hack. ex Matsum. in Bot. Mag. Tokyo 11 (1897) 445, nom. nud.

Nom. Jap. Miyama-nakabo.

Hab. Kuriles austr. Yezo, Hondo, Kiushiu, Korea (Ins. Quelpaert), Shikoku.

forma vivipara Honda in Bot. Mag. Tokyo 51 (1937) 58.

Nom. Jap. Komochi-miyamanukabo.

Hab. Hondo media in montibus.

var. Trinii (Turcz.) Oнwi, comb. nov.

Agrostis Trinii Turcz. Fl. Baic. Dahur. 11. 1299 in Bull. Soc. Nat. Mose. $29: 1$ (1856) 18, nom. provisor.; Litwin. in Sched. Herb. Fl. Ross. 5 (1905) 18; Hult. Fl. Kamtch. 1 (1927) 98; Honda, Monogr. Poac. Jap. (1930) 194 ; Schischk. in Komar. Fl. URSS. 2 (1934) 175; Kitag. Lineam. Fl. Manshur. (1939) 59.

Agrostis canina (non Linn.) Honda, Monogr. Poac. Japon. (1930) 188, p.p.

A typo differt, foliorum laminis subtus superne scabris vel scabriusculis nee laevibus.

Nom. Jap. Kuro-nukabo, Oh-miyama-nukabo.

Hab. Kuriles bor., Saghalien, Korea bor. Distr. Dahuria, Amuria, Okhotsk, Kamtschatka.

Sp. 9. Agrostis canina Linn. Sp. Pl. (1753) 62; Steud. Sym. Gl. 1 (1854) 165; Aschers. et Graebn. Synops. $2: 1$ (1899) 183; Honda, Monogr. Poac. Japon. (1930) 188, partim; Schischk. in Komar. Fl. URSS. 2 (1934\} 
174; Hitche. Man. Grass. U. S. (1935) 340 et 780 ; Philipps. in Journ. Linn. Soc. 51 (1937) 80.

Nom. Jap. Hime-nukabo.

Hab. Japonica, forsan non spontanea (Kiushiu et Hondo rarissima, et Korea). Distr. Reg. bor. omn. part.

var. mutica Gaud. Fl. Helv. 1 (1828) 172; Aschers. et Graebs. Synops. 2:1 (1899) 186; НACK. in Bull. Herb. Boiss. 2:3 (1903) 502; Honda in Bot. Mag. Tokyo 40 (1926) 323 et Monogr. (1930) 189, partim. Nom. Jap. Chosen-nukabo.

Hab. Korea (spontanea), Hondo (?). Distr. Reg. bor. omn. part.

var. formosana HACK. in Bull. Herb. Boiss. $2: 4$ (1904) 528; Matsum. Ind. Pl. Japon. $2: 1$ (1905) 34.

Agrostis transmorrisonensis Hayata, Icon. Pl. Formos. 7 (1918) 84. Agrostis sozanensis Hayata, ibid. (1918) 85; Honda, Mon. (1930) 194. Agrostis canina var. mutica (non Gaud.) Honda, Monogr. (1930) 189 p.p.

Nom. Jap. Sozan-nukabo, Niitaka-nukabo.

Hab. Formosa. Distr. China.

Sp. 10. Agrostis Fukuyamae Онwi in Fedde Repert. 36 (1934) 39.

Nom. Jap. Kumoma-nukabo.

Hab. Formosa, in montibus altissimis.

Sp. 11. Agrostis borealis Har'r m. Handb. Skand. Fl. ed. 3 (1838) 77 ; Steud. Synops. 1 (1854) 166; Aschers. et Graebn. Synops. $2: 1$ (1899) 190; Hılt. Fl. Kamtch. 1 (1927) 94 , OHwi in Act. Phyt. et Geob. 2 (1933) 161; Schischk. in Komar. Fl. URSS. 174; Hitchc. Man. Gr. U. S. (1935) 340 et 779 .

Agrostis viridissima Komar. in Fedde Repert. 13 (1914) 85.

Nom. Jap. Ko-miyamanukabo.

Hab. Kuriles boreal., Yezo, Hondo med. et bor. in alpibus. Distr. Reg. bor. omn. part.

Sp. 12. Agrostis formosana OHwi, sp. nov.

Perennis dense caespitosa estolonifera glabra $20-30 \mathrm{~cm}$ alta, culmis inferne 2-3-nodis laevibus tenuibus erectis, basi breviascendentibus, foliis flaccidis, radicalibus angustissimis convolutis $1 / 2-1 \mathrm{~mm}$ latis, culmeis planis 1-2 mm latis $3-5 \mathrm{~cm}$ longis, ligula brevi obtusa glabra $0.5-1 \mathrm{~mm}$ longa, vaginis laevibus, panicula exserta erecta $5-10 \mathrm{~cm}$ longa, demum effusa laxa ovata, ramis 2-5-nis demum patentibus scabris a medio ramulosis, ramulis patentibus non flexuosis, verticillis basi nudis, spiculis $1.2-1.5 \mathrm{~mm}$ longis pedicellatis viridulis et pallide sordide purpureo-suffusis, glumis aequalibus lanceolato-oblongis acutiusculis univervis, dorso punctulato-asperulis, carina 
fere a basi scabra, lemmate paulo breviore elliptico obtusissimo obsolete 5-nervi mutico, callo glabro, palea $1 / 4-3 / 5$ lemmatis aequilonga ovata, antheris $0.4 \mathrm{~mm}$ longis.

Nom. Jap. Taiwan-yamanukabo.

Hab. Formosa in montibus; m. Taiheizan in Taihokushu (J. Orw n. 2372, typus).

Sp. 13. Agrostis morrisonensis Hayata, Icon. Fl. Formos. 7 (1918) 86 .

Agrostis Clarkei (vix Hook. f.) Hayata in Bot. Mag. Tokyo 21 (1907) 52.

Agrostis flaccida var. morrisonensis (Hayata) Honda in Bot. Mag. Tokyo 40 (1926) 324 et Monogr. Poac. Japon. (1930) 190.

Nom. Jap. Niitaka-nukabo.

Hab. Formosa in montibus altis.

Sp. 14. Agrostis hiemalis (Walt.) Britt. Stern, et Poggenb. Prel. Cat. N. Y. (1888) 68; Kordz. Pl. Sachal. Nakah. (1910) 17 ; Hult. Fl. Kamtch. 1 (1927) 97 ; Honda, Monogr. Poac. Japon. (1930) 190 ; Schischk. in Komar. Fl. URSS. 2 (1934) 186 ; Hiтchc. Man. Gr. U. S. (1935) 337 et 781.

Cornucopiae hiemalis WALT. Fl. Carol. (1788) 73.

Agrostis scabra Willd. Sp. Pl. 1 (1797) 370 ; Hack. in Bull. Herb. Boiss. 7 (1899) 648 et $2: 3$ (1903) 502 ; Fernald in Rhodora 35 (1933) 207. Agrostis laxiflora R. Br. in Richard. Bot. App. Fankl. Journ. 1(1823) 3. Nom. Jap. Yezo-nukabo.

Hab. Kuriles, Saghal. Yezo, Hondo med. et bor. Distr. Amer. bor., As. or.

Sp. 15. Agrostis clavata Trin. in Spreng. Neue Entd. 2 (1821) 55; Hult. Fl. Kamtch. 1 (1927) 95; Honda, Monogr. Poac. Japon. (1930) 194.

?Agrostis grandis (non Trin.) Honda, l. c. (1930) 190.

Agrostis perennans (non Tuckerm.) Auct. fl. Japon. etiam Honda, l.c. 193.

Agrostis Scouleri (non Trin.) Hack. in Bull. Herb. Boiss. 2:4 (1904) 523.

Agrostis Matsumurae Hack. ex Matsum. in Bot. Mag. Tokyo 11 (1897) 445 nom. nud.; Honda, Monogr. Poac. Japon. (1930) 191, p.p. maxim.

Agrostis tenuiflora (non Willd.) Steud. Syn. 1 (1854) 163; Hack. in Bull. Herb. Boiss. 7 (1899) 648.

Agrostis valvata Steud. Synops. 1 (1854) 171.

Agrostis macrothyrsa Hack. in Fedde Repert. 7 (1909) 318.

Nom. Jap. Yama-nukabo. 
Hab. In Japponia tota in locis montosis communis. Distr. Asia bor. et Europ. bor-orient.

var. Nukabo, OHwi, var. nov.

Agrostis Matsumurae (vix Hack.) Matsum. l.c. p.p.; Honda, l.c. p.p.

A typo panicula etiam anthesi contracta, verticillis a basi spiculosis differt. Typus: Kyoto, leg. J. Oнwi, n. 9181.

Nom. Jap. Nukabo.

Hab. In Japonia tota in pratis communis. Distr. China.

Sp. 16. Agrostis exarata Trin. Diss. 1, De Gram. Unifl. et Sesquif!. (1824) 207; Hult. Fl. Kamtch. 1 (1927) 96; Schischk. in Komar. Fl. URSS. 2 (1934) 186; HitchC. Man. Grass. U. S. (1935) 335 et 780.

Agrostis grandis Trin. in Mém. Acad. Pétersb. 6, Sci. Nat. $4: 1$ (1841) 316.

Agrostis densiflora (non Vasey) Honda, Monogr. Poac. Jap. (1930) 189 .

Nom. Jap. Chishima-nukabo.

Hab. Kuriles boreal. Distr. Americ. bor. et Kamtch.

Sp. 17. Agrostis arisan-montana Orwi in Act. Phytotax. et Geobot. 2 (1933) 161.

Nom. .Jap. Arisan-nukabo.

Hab. Formosa.

Sp. 18. Agrostis nipponensis Honda in Bot. Mag. Tokyo 41 (1927) 380 et Monogr. Poac. Japon. (1930) 187.

Nom. Jap. Hime-konukagusa.

Hab. Hondo, Shikoku, Kiushiu, in locis humidis rarius occurrit.

Gen. 2. Polypogon Desf. Fl. Atl. 1 (1798) 66.

1. (Ylumae inter lobos arista quam gluma "2-3-plo longiore praeditae, spiculae basi callo hrevi latiusculo subobconico praeditae.

P. monspeliensis.

1. Clumae inter lobos vel ex apice arista quam gluma aequilonga vel breviore praeditae, spiculae basi callo tenui longiusculo praeditae.

2. Glumae inter lobos minutos aristatae, arista quam gluma subaequilonga vel paullo t) reviore.

P. fugax.

2. Glumae integrae, ex apice arista multo breviore praeditae.

P. hondoensis.

Sp. 19. Polypogon monspeliensis (Linn.) Desf. Fl. Atl. 1 (1798) 67 ; A. Gray, Bot. Japan (1859) 328; Aschers. et Graebn. Synops. $2: 1$ (1899) 160 ; Rendle in Journ. Linn. Soc. 36 (1904) 386 ; Honda, Monogr. (1930) 197 ; Roshev. in Komar, Fl. URSS. 2 (1934) 164; Hitchc. Man. Grass. U. S. (1935) 350 et 937.

Alopccurus monspeliensis LinN. Sp. Pl. (1753) 61. 
Nom. Jap. Hama-hiegaeri.

Hab. Hondo, Shikoku, Kiushiu, Riukiu, Formosa, Korea. Distr. Reg. bor. omn. part.

Sp. 20. Polypogon fugax Steud. Synops. 1 (1854) 184 ; C. E. Hubb. in Prain, Fl. Trop. Afr. 10 (1937) 162.

Polypogon Higegaweri Steud. 1. c. (1854) 422; Honda, Monogr. Poac. Japon. (1930) 198; Mryabe et Kudo, Fl. Hokk. a. Saghal. 2 (1931) 132.

Polypogon demissus Streud. Synops. 1 (1854) 422 ; Roshev. in Komar. Fl. URSS. 2 (1934) 164.

Polypogon littoralis (non Smith) A. Gray, Dr. Pl. Coll. Jap. (1856) 328 ; Ноок. f. Fl. Brit. Ind. 7 (1897) 246 (var. Higegaweri Hook. f.); Rendle in Journ. Linn. Soc. 36 (1904) 386.

Polypogon miser Makino in Bot. Mag. Tokyo 26 (1912) excl. syn.

Nom. Jap. Hiegaeri.

Hab. Hondo, Shikoku, Kiushiu, Riukiu, Fỏrmosa, Korea. Distr. China, Nepaul, Africa.

Sp. 21. Polypogon hondoensis OHwi, sp. vel hybr. nov.

Agrostis hondoensis $\mathrm{OHwI}$, hoc loc.

?Polypogon fugax $\times$ Agrostis palustris.

Annua? basi longe repens $20-40 \mathrm{~cm}$ alta, culmis laevibus sursum erectis, foliorum laminis planis membranaceis cinereovirentibus $3-5 \mathrm{~mm}$ latis $5-10 \mathrm{~cm}$ longis scabriusculis, ligula $3-4 \mathrm{~mm}$ longa glabra, vaginis internodia plerumque superantibus laevibus, panicula oblongo-ovata dense sed genere laxiuscule spiculosa $5-8 \mathrm{~cm}$ longa $2-2.5 \mathrm{~cm}$ lata, ramis ascendentibus brevibus semiverticillatis scabris basi breviter nudis, verticillis a basi spiculiferis, pedicello clavato-incrassato, spiculis $2 \mathrm{~mm}$ longis plus minus purpurascentibus, glumis requalibus late lanceolatis dorso scabris, apice acuto integro brevissime aristatis, arista $1 / 4^{-1 / 8}$ glumae aequante, lemmate ovato pellucido membranaceo $1.5 \mathrm{~mm}$ longo glabro obsoletissime 5-nervi truncato 4-denticulato, nervo medio ex apice excurrente mucronato, palea paullo breviore 2 -nervi, callo glabro, rhachilla 0 , staminibus 3 , antheris $2 / 3 \mathrm{~mm}$ longis, ovario glabro.-A P. fugaci glumis integris acutis brevissime aristatis differt.

Nom. Jap. Nukago-gaeri.

Hab. Hondo (Omagoe in Mutsu, leg. S. Muramatsu n. 170).

Gen. 3. Phleum Linn. Sp. Pl. (1753) 59 et Gen. ed. 5 (1754) 29.

1. Rhizomatosa, glumae membranaceae, carina recta longe patentim ciliata, apice excurrens in aristam $1 / 2-4 / 5$ glumae aequilongam, rhachilla ultra florem non producta.

2. Spica ovata vel oblonga, $1.5-3 \mathrm{~cm}$ longa.

P. alpinum.

2. Spica cylindrica, $3-15 \mathrm{~cm}$ longa.

P. pratanse.

1. Erhizomatosa, annua, spica cylindrica angusta, glumae coriaceae flavovirentes, 1/2- 
obovatale, dorso superne inflatie, glabrae vel saepius in earina brevi-patule-ciliatae, apice cuspidatae.

P. paniculatum.

Sp. 22. Phleum alpinum Lins. Sp. Pl. (1753) 59; Aschers. et Graebs. Synops. $2: 1$ (1898) 144 (P. pratense subsp.) ; Hack. in Bull. Herb. Boiss. 7 (1899) 647 et $2: 4$ (1904) 523; Honda, Monogr. (1930) 207 ; Mryabe et Kuno, Fl. Hokk. a. Saghal. 2 (1931) 131; Roshev. in Komar. Fl. URSS. 2 (1934) 135; Нrтснс. Man. Grass. U. S. (1935) 353 et 927.

Nom. Jap. Miyama-awagaeri.

Hab. Kuriles bor., Yezo, Hondo media in alpibus, Korea (ficle Honda) Formosa in montibus. Distr. Reg. arct. et alpin.

Sp. 23. Phleum pratense Linn. Sp. Pl. (1753) 59; Hack. in Bull. Herb. Boiss. 7 (1899) 647; Aschers. et Graebs. Synops. $2: 1$ (1899) 141 (subsp. vulgare); Honda, Monogr. (1930) 208 ; Miyabe et Kudo, Fl. Hokk. a. Saghal. 2 (1931) 132; Roshev. in Komar. Fl. URSS. 2 (1934) 132 ; Hitchc. l.c. (1935) 353 et 927; Kitag. Lineam. Fl. Manshur. (1939) 86.

Nom. Jap. O-awagaeri.

Hab. In Japonia late dispersa, sed non spontanea. Distr. Europ.

Sp. 24. Phleum paniculatum Huds. Fl. Angl. (1762) 23 et 26. var. annuum (M. v. Bieb.) Honda, Mon. Poac. Japon. (1930) 208.

Phleum annuum M. v. Bieb. Fl. Taur. Caucas. 1 (1806) 46.

Phleum asperum JACQ. var. annuum (M. v. Bieb.) Grisb. in Ledeb. Fl. Ross. 4 (1854) 456 ; Hack. in Bull. Herb. Boiss. 7 (1899) 647.

Phleum japonicum Franch. et Savat. Enum. Pl. Japon. 2 (1879) 593.

Phleum asporum var. japonicum (Franch. et SAvat.) Hack. ex Matsu n. in Bot. Mag. Tokyo 11 (1897) 444 et in Bull. Herb. Boiss. 7 (1899) 647.

Nom. Jap. Awagaeri.

Hab. Hondo, Shikoku, Kiushiu. Distr. China, Siberia oceid.

Gen. 4. Alopecurus Linn. Sp. Pl. (1753) 60 et Gen. ed. 5 (1754) 30.

1. Spica globosa vel oblonga usque ad $3 \mathrm{~cm}$ longa, 10-15 $\mathrm{mm}$ lata, dense lanata, glumale quam lemma longiores, rhizoma breviter repens. ............... Stejnegeri.

1. Spica cylindrica non lanata, fere glabra vel pilosil, $4-10 \mathrm{~cm}$ longa, glumae quam lemma aequilongae vel subbreviores.

2. Spiculae $4-7 \mathrm{~mm}$ longae.

3. Annua estolonifera, spiculae $6-7 \mathrm{~mm}$ longae, duriuscule menulranaceac, spira densiuscula.

4. Glumae liberae vel hasi tantum comnatae, antherae pallide flavescentes.

4. Glumae ad vel ultra medium usque connatae, antherae flavescentes.

A. japonicus.

A. myosuroides.

3. Perennis, brevi-stolonifera, spicula 4-.) mm longa pallida vel pallide virens interdum purpureo-suffusa mollis, antherae saturate flavae $2.5-3 \mathrm{~mm}$ longae, spica densissima. 
2. Spiculae 2-2.5 mm longae, antherae aurantiacae $0.6-1 \mathrm{~mm}$ longae. .... A. aequalis.

Sp. 25. Alopecurus Stejnegeri Vasey in Proc. U. S. Nat. Mus. 10 (1887) 153; Komar. Fl. Pen. Kamtsch. 1 (1927) 136; Roshev. in Komar. FI. URSS. 2 (1934) 156.

Alopecurus alpinus var. borealis (non GrIseb.) Kudo, Fl. Paramushir (1922) 67.

Alopecurus alpinus car. Stejnegeri (VASEy) Hult. Fl. Kamtch. 1 (1927) 90; Honda, Monogr. Poac. Japon. (1930) 204; Miyabe et Kudo, Fl. Hokk. a. Saghal. 2 (1931) 131.

Nom. Jap. Chishima-yarikusa.

Hab. Kuriles boreal. Distr. Reg. Behringii.

Sp. 26. Alopecurus japonicus Steud. Synops. 1 (1854) 149 ; Hack. in Bull. Herb. Boiss. 7 (1899) 648; Rendle in Journ. Linn. Soc. 36 (1904) 385 ; Honda, Monogr. (1930) 205.

Alopecurus malacostachyus A. Gray, in Perry Exped. Jap. Bot. 2 (1856) 328.

Nom. Jap. Seto-gaya.

Iab. Hondo, Kiushiu. Distr. China media.

Sp. 27. Alopecurus myosuroides Huds. Fl. Angl. (1762) 23; Aschers. et Graebn. Synops. $2: 1$ (1899) 130; Hitchc. Man. Grass. U. S. (1935) 347 et 788.

Alopecurus agrestis Linn. Sp. PI. ed. 2 (1762) 89; Rendle in Journ. Linn. Soc. 36 (1904) 385; Hayata in Bot. Mag. Tokyo 21 (1907) 55 et Icon. Pl. Formos. 7 (1918) 82 ; Honda, Monogr. (1930) 203.

Nom. Jap. No-suzumenoteppo.

Hab. Formosa, fide Hayata et Honda, l. c. Distr. Asia et Europ.

Sp. 28. Alopecurus pratensis IJnN. Sp. Pl. (1763) 60 ; Aschers. et GraebN. Synops. $2: 1^{\circ}$ (1899) 131; Hack. in Bull. Herb. Boiss. 7(1899) 648 ; Honda, Monogr. (1930) 206 ; Rosinev. in Komar. Fl. URSS. 2 (1934) 150 ; Hitchc. Man. Grass. U. S. (1935) 347 ; Kiragawa, Lineam. Fl. Manshur. (1939) 60.

Nom. Jap. O-suzumenoteppo.

Hab. In Japonia (Hondo, Shikoku, et Yezo) introducta. Distr. Europ.

Sp. 29. Alopecurus aequalis Sobol. Fl. Petrop. (1799) 16; Rendle in Journ. Linn. Soc. 36 (1904) 384 ; Roshev. in Komar. Fl. URSS. 2 (1934) 158 ; Hiтchc. Man. Grass. U. S. (1935) 348 et 787.

Alopecurus geniculatus (non LinN.) Auct. fl. Jap. etiam Hond, Monogr. (1930) 204.

Alopecurus fulvus J. E. Sмiтh, in Smith et Sowerb. Engl. Bot. 21 
(1805) t. 1467 ; Fr. Schm. Reis. (1868) 203; Aschers. et Graebn. Synops. $2: 1(1899) 137$.

Nom. Jap. Kita-suzumenoteppo.

Hab. Kuriles boreal., ?Saghalien. Distr. Reg. bor.

var. amurensis (Komar.) Ohwi, comb. nov.

Alopecurus fulvus var. amurensis Komar. Fl. Manshur. in Act. Hort. Petrop. 20 (1901) 272 in nota.

Alopecurus geniculatus var. amurensis (Komar.) Roshev. in Bull. Jard. Bot. Pierre Gr. 14, suppl. (1914) 54.

Alopecurus amurensis (Komar.) Komar. in Bull. Jard. Bot. 16 (1916) 151; Roshev. in Komar. Fl. URSS. 2 (1934) 158; Kitag. Lineam. Fl. Manchur. (1939) 59.

Nom. Jap. Suzumeno-teppo.

Hab. Saghalien, Yezo, Hondo, Shikoku, Kiushiu, Riukiu, Formosa, Korea. Distr. China, Manchuria, Sibiria orientalis.

var. brachytrichus (OHwi) OHwi, comb. nov.

Alopecurus brachytrichus Онші in Act. Phytot. et Geob. 5 (1936) 51.

Nom. Jap. Yama-suzumenoteppo.

Hab. Korea borealis, in locis humidis montanis.

Trib. 2. Cinneae OHwi ${ }^{*}$

Gen. 5. Cinna Linn. Sp. Pl. (1753) 5 et Gen. ed. 5 (1754) 6.

Sp. 30. Cinna latifolia (Trevir.) Griseb. in Ledeb. Fl. Ross. 4 (1853) 435 ; Fr. Sch m. Reis. (1868) 203 ; Honda, Monogr. Poac. Japon. (1930) 196; Rosinev. in Komar. Fl. URSS. 2 (1934) 169 ; Hıтchc. Man. Grass. U. S. (1935) 344 et 827 ; Kitag. Lineam. Fl. Manchur. (1939) 68.

Agrostis latifolia Trevir. in Goepp. Beschr. Bot. Gart. Bresl. (1830) 82.

Muhlenbergia pendula Trin. in Bong. Veg. Sitcha (1832) 172.

Cinna pendula (Trin.) Trin. in Mém. Acad. Pétersb. 6, Sci. Nat. 4:1 (1841) 280 ; Steud. Synops. 1 (1854) 182 ; Aschers. et Graebn. Synøps. $2: 1$ (1899) 159 .

Agrostis alba var. koreensis NakAI in Bot. Mag. Tokyo 33 (1919) 1.

Nom. Jap. Fusa-gaya.

Hab. Saghalien, Yezo, Hondo bor. med. 1 Korea. Distr. Reg. subarct.

*) Trib. Cinneae OHwi, trib. nov.-Spiculae 1-florae paniculatae a latere compressae, rhachilla ultra florem non vel breviter producta, glumis 2 inaequalibus vel subaequalibus spiculas non vel vix superantibus, lemmate compresso carinato viridulo $\overline{5}$-nervi vel nervis intermediis obsoletis subtrinervi mutico vel sub apice brevimucronato, palea subbreviore, carinis 2 approximatis vel connatis, stylis brevibus, stigmatibus plumosis albidis, caryopside oblonga libera tereti glabra.-Gramina elata foliis planis, panicula saepe effusa viridula. Typus: Cinna LinN. 
Gen. 6. Aulacolepis Hack. in Fedde Repert. 3 (1907) 241.

1. Gluma $1^{m a}$ minuta $1 / 3$ glum. $2^{\text {dim }}$ aequans vel brevior $0.5 \mathrm{~mm}$ longa vel brevior, foliorum

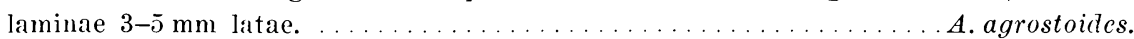

1. Gluma $1^{\mathrm{ma}}$ dimidium $\mathrm{gl}$. $2^{\text {dae }}$ a equans, $1-1.5 \mathrm{~mm}$ longa, foliorum laminae $7-15 \mathrm{~mm}$ latae.

A. Treutleri.

Sp. 31. Aulacolepis agrostoides (Merr.) Ohwi in Act. Phytot. et Geobot. 4 (19:35) 30.

Aniselytron agrostoides Merr. in Philipp. Journ. Sci. 5 (1910) Bot. 329. var. formosana Oriwı, l. c. (1935) 30.

Nom. Jap. Ao-konukagusa.

Hab. Formosa. Distr. Philippine (Spec.).

Sp. 32. Aulacolepis Treutleri (O. Kuntze) Hack. l. e. (1907) 242; Ionda, Monogr. Poac. Japon. (1930) 195.

Milium Treutleri O. Kuntze, Revis. Gen. Pl. 2 (1891) 780, ex pte.

Deyeuxia Treutleri (O Kuntze) Stapf in Hook. Icon. 24 (1895) t. 2396.

Poa milioides Honda in Bot. Mag. Tokyo 41 (1927) 161.

Aulucolepis milioides (Honda) Orwi in Act. Phytotax. et Geobot. 2 (1933) 161.

Aulacolepis Treutleri var. milioides (Honda) OHwi, ibid. 6 (1937) 151.

Nom. Jap. Kogome-ichigotsunagi.

Hab. Formosa. Distr. Sikkim· Himalava, China austr.-oceid.

var. japonica (HAck.) OHwi, comb. nov.

Aulacolepis japonica Hack. l. c. (1907) 241; Koidz. in Matsum. Icon. Pl. Koishikav. 4 (1918) 13, t. 219 ; Honda, Monogr. (1930) 195.

Nom. Jap. Hirohano-konukagusa.

Hab. Hondo occid.

Trib. 3. Phaenospermeae Roshev. Zlaki (1937) 242.

Gen. 7. Phaenosperma Munro ex Benth in Journ. Linn. Soc. 19 (1881) 59 .

Sp. 33. Phaenosperma globosa Munro ex Benth, l. c. (1881) 59 ; Hook. Icon. Pl. (1891) t. 1991; Makino in Bot. Mag. Tokvo 17 (1903) 209 et 18 (1904) 222 in japonice; RendLe in Journ. Linn. Soc. 36 (1904) 340; Honda, Monogr. Poac. Japon. (1930) 313 .

Nom. Jap. Takikibi, Kashima-gaya, O-tatsunohige.

Hab. Hondo occid., Shikoku, Kiushiu, Korea austr., Formosa. Distr. China.

Trib. 4. Brachyelytreae OHwi. ${ }^{*}$

*) Trib. Brachelytreae OHwi, trib. nov.-Spiculae angustae 1-florae, rhachilla ultra florem longe producta sub flore articulata, (allo obtuso piloso-scabro, glumis 2 
Gen. 8. Brachyelytrum Beauy. Ess. Agrost. (1812) 39.

Sp. 34. Brachyelytrum japonicum (НАск.) Наск. еx Matsum. in Bol. Mag. Tokyo 11 (1897) 444, nom. tant.; HondA, Monogr. Poac. Japon. (1930) 210.

Brachyclytrum crectum var. japonicum HACK. in Bull. Herb. Boiss. 7 (1899) 647.

Nom. Jap. Koya-zasa.

Hab. Hondo, Shikoku, Kiushiu, Korea (Ins. Quelpaert).

\section{のがりやす屬ヨ除イタ邦産ぬかぼ族（和交摘要）}

大井次三毁

從來, Agrosteae (Agrostideae) カラ STAPF, C. E. Hubbard, Rosheviry 等ニョッ テ Sporoboleue, Stipeae ガ除外サレタノデソレ等八此所デハ論及シナイ。又 Phaenosperma 八Iristegineae ヤ Arundinelleae 二入レラレルガ，花部ノ構造ハ十イ意 味ノAgrosteae ソノモノデアルカラ此所二付加へ夕。Agrostis 、Agrosteae つ中 ヨナシ，小怔中ノ花數ヨ考虑シナケレバ Culamagrostis, Polypogon ト共= Aveneate トノ差ガ多ドナイ。此ト同ジ關係八廣義ノAgrosteae F Festuceat 八群ノ間二モ 兒ラレル。此レヨ本邦產ノ小群ノミニツイテ苂示スルト，

\begin{tabular}{|c|c|c|}
\hline $2-\infty$ 小花/群 & 特 & 1 小花，群 \\
\hline $\begin{array}{l}\text { Festuceae- } \\
\text {-Brominale }\end{array}$ & 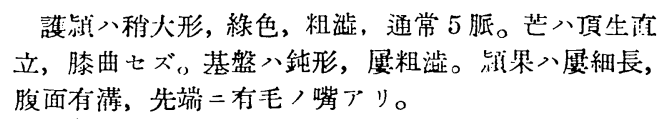 & Brachyelytreac \\
\hline $\begin{array}{l}\text { Festuceae- } \\
\text {-F'estucinae }\end{array}$ & 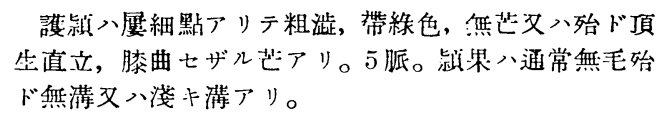 & Cinneae \\
\hline $\begin{array}{l}\text { Festuceae- } \\
\quad \text {-Diarrheninae }\end{array}$ & 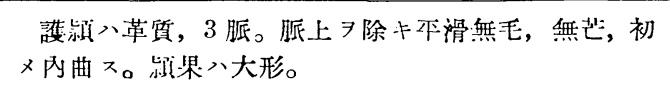 & $\begin{array}{l}\text { Phaeno- } \\
\text { spermeale }\end{array}$ \\
\hline Areneae & 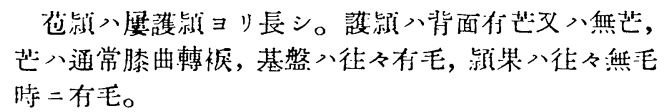 & $\begin{array}{l}\text { Agrosteae } \\
\text { (sens. str.) }\end{array}$ \\
\hline
\end{tabular}

ソシテ大體トシテ左ノ群カラ右ノ群ガ進化シタト考へラレルカラ，本邦産ノ從來 , Agrosteue Э歐文欄登裁ノ樣二細分シタイ。Calamagrostis ᄏ除イタ占イ(廣イ) 意味,Agrosteue 八本邦二八 8 屬 34 種ガ認メラレル。份 Alopecurus ノ類緣關 係八疑問デ Phalarideae 二近イ所モアルガ通說ニ從ツ夕。

brevibus, lemmate angusto 5 -nervi rigido acuminato scabro rectiaristato, palea bieostata, stylis distinctis, stigmatibus elongatis plumosis allidis, ovario oblongo-lanceolato, superne pilosello, caryopside lineari-oblonga libera ventre profunde sulcata, vertice rostro albido pilossello terminata.-Gramen sylvaticum, foliis planis, panicula angusta. Typus: Brachyelytrum BEAUv. 